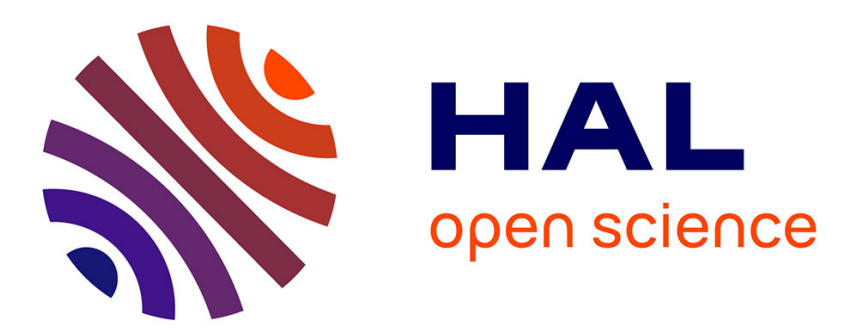

\title{
High resolution and wideband integrated optics infrared stationary-wave spectrometer fabricated by ultrafast laser inscription
}

\author{
Irene Heras, Javier Vázquez de Aldana, Alain Morand, Guillermo Martin
}

\section{To cite this version:}

Irene Heras, Javier Vázquez de Aldana, Alain Morand, Guillermo Martin. High resolution and wideband integrated optics infrared stationary-wave spectrometer fabricated by ultrafast laser inscription. SPIE Astronomical Telescopes + Instrumentation, Jun 2018, Austin, United States. 10.1117/12.2311440 . hal-01961059

\section{HAL Id: hal-01961059 \\ https://hal.science/hal-01961059}

Submitted on 22 Jan 2021

HAL is a multi-disciplinary open access archive for the deposit and dissemination of scientific research documents, whether they are published or not. The documents may come from teaching and research institutions in France or abroad, or from public or private research centers.
L'archive ouverte pluridisciplinaire HAL, est destinée au dépôt et à la diffusion de documents scientifiques de niveau recherche, publiés ou non, émanant des établissements d'enseignement et de recherche français ou étrangers, des laboratoires publics ou privés. 


\section{High resolution and wideband integrated optics infrared stationary- wave spectrometer fabricated by ultrafast laser inscription}

I. Heras, J. R. Vázquez de Aldana, A. Morand, G. Martin

I. Heras, J. R. Vázquez de Aldana, A. Morand, G. Martin, "High resolution and wideband integrated optics infrared stationary-wave spectrometer fabricated by ultrafast laser inscription," Proc. SPIE 10706, Advances in Optical and Mechanical Technologies for Telescopes and Instrumentation III, 107063A (10 July 2018); doi: 10.1117/12.2311440

Event: SPIE Astronomical Telescopes + Instrumentation, 2018, Austin, Texas, United States 


\title{
High resolution and wideband integrated optics infrared stationary- wave spectrometer fabricated by ultrafast laser inscription
}

\author{
I. Heras ${ }^{\mathrm{a}}$, J.R.Vazquez de Aldana ${ }^{\mathrm{b}}$, A. Morand ${ }^{\mathrm{c}}$ and G. Martin ${ }^{\mathrm{a}}$ \\ ${ }^{a}$ Univ. Grenoble Alpes, CNRS, IPAG, F-38000 Grenoble, France \\ ${ }^{\mathrm{b}}$ Universidad de Salamanca, 37008 Salamanca, Spain \\ ${ }^{\mathrm{c}}$ IMEP-LAHC, Minatec, 3 Parvis Louis Néel, CS 50257, 38016 Grenoble, France
}

\begin{abstract}
Direct laser writing is a powerful technique for the development of astrophotonic devices, namely by allowing 3D structuring of waveguides and avoiding in-plane crossings that can induce losses and crosstalk in multi-telescope beam combiners. In this work, a multiplexed device is proposed in order to increase the spectral bandwidth to hundreds of $\mathrm{nm}$, for a central wavelength of $1580 \mathrm{~nm}$. Our device is fabricated by ultrafast laser inscription of type I waveguides in bulk IR-grade fused silica glass. A first part of the study was devoted to finding the optical fabrication parameters in terms of depth, speed and number of tracks needed to achieve an optimal waveguide, single mode in the near IR. A second part was focused to the fabrication of different optical lanterns, from one multimode input to several (4 or 16) single mode outputs. The optical chip consists of a multimode input slit-waveguide, that adiabatically converts into an aligned matrix of 4 or 16 single-mode channel waveguides, with a pitch corresponding to the detector pixel size. Two separations $(20 \mu \mathrm{m}$ and $64 \mu \mathrm{m})$ were studied, in order to avoid crosstalk between parallel waveguides (directional coupling) and from extracted flux into the detector (pixel crosstalk). A final part is dedicated to the spectrometer realization, based on the sampling of a stationary wave inside the waveguide.
\end{abstract}

Keywords: integrated optics, visible interferometry, electro-optic modulation, Lithium Niobate, glass waveguides, direct laser writing.

*guillermo.martin@univ-grenoble-alpes.fr; phone +33 47663 52 76; www. ipag.osug.fr

\section{INTRODUCTION}

It is well known that integrated optics beam combiners are a good approach to reduce problems of stability, weight and compacity of interferometric instruments. Moreover, using single mode optical waveguides it is possible to achieve modal filtering, a compulsory stage for increasing fringe contrast in high contrast interferometry applications. Secondly, electro-optic materials, allow to modify the refractive index on chip and at high frequencies (above 100kHz), opening applications for phase and amplitude modulation. Among the different techniques available for waveguide fabrication, in recent years there has been an increasing interest in direct laser writing of waveguides [1-3], as a versatile tool for modifying the refractive index as needed, and playing with the vertical direction, such that in-plane X-crossing of waveguides is avoided, reducing crosstalk and increasing the compacity of the device. Thus, in the context of multitelescope interferometry, it becomes possible to conceive a beam combiner using more than 6 telescopes, and fabricate the whole combination system in a single wafer.

In this paper we present the first realizations in a borosilicate glass of direct laser written waveguides and lanterns, devoted to high resolution integrated spectrometers. The paper is organized as follows: in a first part we study the different parameters that allow for single mode operation in the near IR, by varying the laser scanning speed, the number of tracks or the depth of photo-inscription. In a second part we recall the principle of operation of a stationary wave 
Fourier transform spectrometer (SWIFTS) in order to stress the drawback of sampling resolution and limited spectral etendue. We therefore propose a multiplexing alternative: SWIFTS-Wide by developing a photonic lantern in our material. Finally, we present our recent developments on coupling the waveguides (where the stationary wave, i.e. the wide band interferogram is sampled) to the detector were the sampled signal is recorded for later Fourier transform analysis.

\section{LASER WRITTEN WAVEGUIDES}

In this study we will focus only in Direct Laser Writing of Type I waveguides [4-6], where the focus spot of the laser is the volume where refractive index increases. The index modification obtained is a function of the material and also of the laser parameters (energy of the pulse, scan speed, number of tracks...). In our case, the typical energies are 5 $\mu \mathrm{J}$, scan speed $25-200 \mu \mathrm{m} / \mathrm{s}$ and we use a multiscan approach. This means that the waveguide is built after several scans of the laser (number of tracks), that are realized close together (see table I). Finally, the depth into the material and the focusing objective are also crucial parameters. In our case, the fabrication of waveguides and lanterns was done in Borosilicate Glass using a x50 objective, at 50 and 100 um depth. The summary of the parameters is presented in table I:

\begin{tabular}{|c|c|c|c|c|c|c|c|}
\hline $\begin{array}{l}\text { Test } \\
\text { № }\end{array}$ & № & $\begin{array}{c}\text { Focus } \\
\text { objective }\end{array}$ & $\begin{array}{l}\text { Scan speed } \\
(\mu \mathrm{m} / \mathrm{seg})\end{array}$ & $\begin{array}{l}\text { Pulse energy } \\
\mu J \text { (read/real) }\end{array}$ & $\begin{array}{l}\text { № tracks / } \\
\text { distance }\end{array}$ & $\begin{array}{l}\text { Depth } \\
(\mu \mathrm{m})\end{array}$ & Comments \\
\hline \multirow{8}{*}{ T3 } & 3.1 & \multirow{8}{*}{$50 x$} & \multirow{8}{*}{100} & \multirow{8}{*}{$0.38 \times(16)$} & $1 / 2 \mu \mathrm{m}$ dist & \multirow{8}{*}{50} & Martes 23/01 \\
\hline & 3.2 & & & & $2 / 2 \mu \mathrm{m}$ dist & & \\
\hline & 3.3 & & & & $3 / 2 \mu \mathrm{m}$ dist & & \\
\hline & 3.4 & & & & $4 / 2 \mu \mathrm{m}$ dist & & \\
\hline & 3.5 & & & & $5 / 2 \mu \mathrm{m}$ dist & & \\
\hline & 3.6 & & & & $6 / 2 \mu \mathrm{m}$ dist & & \\
\hline & 3.7 & & & & $7 / 2 \mu \mathrm{m}$ dist & & \\
\hline & 3.8 & & & & $8 / 2 \mu \mathrm{m}$ dist & & \\
\hline
\end{tabular}

Table I: Number of waveguides (8) fabricated using incremental number of tracks.

Similar studies were achieved for scanning speed, depth and laser energy. No big differences were observed between 80 and $100 \mu \mathrm{m} / \mathrm{sec}$ writing speed. We have selected $50 \mu \mathrm{m}$ depth, 4 tracks multiscan and $100 \mu \mathrm{m} / \mathrm{sec}$ as is a good compromise between writing speed and properties of the guiding mode. The image of the incremental waveguides, as well as the mode field for a particular 4 tracks waveguide at $\lambda=1580 \mathrm{~nm}$ is shown in figure 1 :

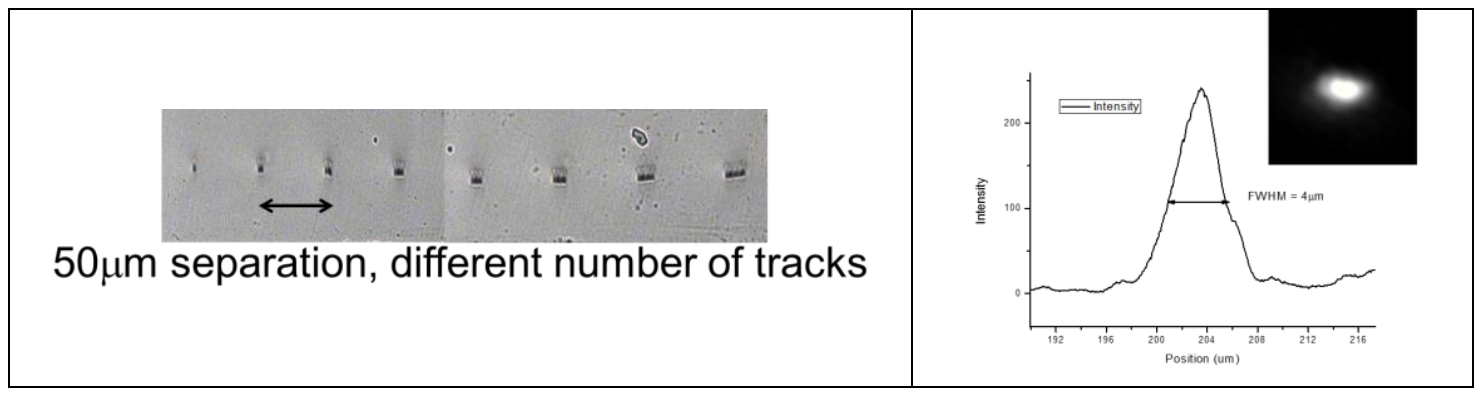

Fig.1: Left: Output of the waveguides, showing the geometry of the type I channel waveguide, as a function of the number of tracks. Right: Mode field observed in the 4 tracks waveguide and typical FWHM at $\lambda=1580 \mathrm{~nm}$. 
The mode obtained for the 4 tracks, $50 \mu \mathrm{m}$ depth and scanning speed of $100 \mu \mathrm{m} / \mathrm{sec}$ shows a FWHM $=4 \mu \mathrm{m}$. We then realized some tests of wideband transmission, using a SLED. As expected, the transmission decreases as the number of tracks is reduced, as seen in figure 2. Measurements at larger spectral range are currently being achieved, in order to get the cut-off wavelength and range of operation of the channel waveguides. We can nonetheless confirm that the parameter used allow for single mode waveguide around $1580 \mathrm{~nm}$.

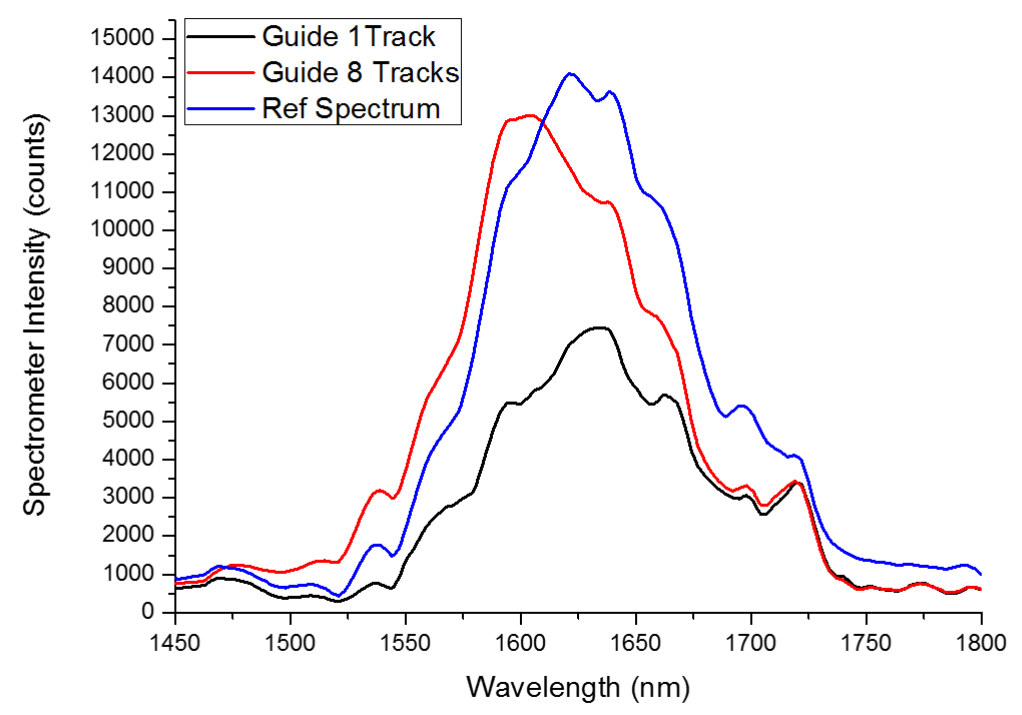

Fig.2: Wideband spectrum of the 1 track and 8 tracks channel waveguides, as compared to the reference spectrum (not to scale). The spectral band is transmitted, but with lower global transmission for the 1 track channel waveguide.

Once we optimized the single mode near IR channel waveguide, we focused on the realization of the multiplex approach to high resolution, high spectral etendue integrated Fourier Transform spectrometer.

\section{HIGH SPECTRAL RESOLUTION INTEGRATED SPECTROMETERS}

Reducing the size and power consumption of instruments has always been an issue for spatial applications. Nowadays, with the development of Nanosat projects, the possibility of achieving very high resolution in a very compact and light device gives waveguide optics a very broad field of application. Our approach is to develop integrated spectrometers where the spectrum is obtained by sampling interference fringes of the source, as proposed in the SWIFTS-Lippmann configuration [7-10]. The stationary wave is obtained by superposition of direct and backward guided beams, the last being obtained by reflection at the output of the channel waveguide. The backward field is naturally occurring by Fresnel reflexion (that can be tens of \% for high index materials such as Ge, chalcogenide glasses or Lithium niobate). Here, as we use a Borosilicate glass, is better to use a mirror coating at the output of the waveguide, in order to achieve high contrast fringes. The principle of operation of the stationary wave interferometer is recalled in figure 3: 


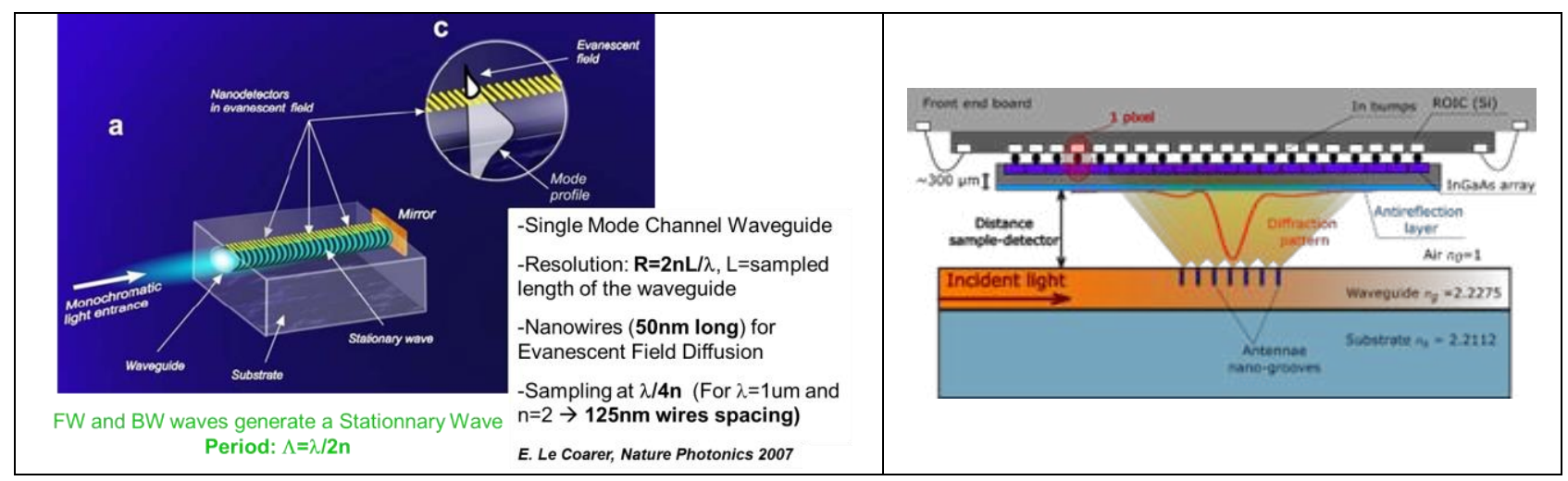

Fig. 3: Left: Principle of operation of a stationary wave Fourier transform spectrometer (SWIFTS), and typical values for Resolution and sampling requirements, to fulfil Shannon-Nyquist constraints. Right: Principle of the compact spectrometer: coupling the scattered signal to a detector, to get as close as possible.

As discussed in previous papers [7,8], to correctly sample the interferogram, one would need sampling centers at least with $\Lambda=\lambda / 4 \mathrm{n}=187 \mathrm{~nm}$ separation at $\lambda=1.5 \mu \mathrm{m}$. Besides, in order to obtain high resolution is compulsory to achieve a sampling length of several millimeters, which is easily done with photo-inscription. Theoretical values for spectral resolution and spectral window, $\Delta \sigma$, are $\mathrm{R}=2 \mathrm{~nL} / \lambda$ and $\Delta \sigma=1 / 4 \mathrm{n} \Delta \mathrm{L}$. With $\mathrm{L}=10000 \mu \mathrm{m}$ (length of the sampling region), $\mathrm{n}=1.5$ the effective refractive index and $\lambda=1.5 \mu \mathrm{m}$, we expect $\mathrm{R}=20000$, which gives an idea of the spectral resolution we can obtain in this simple device. The spectral window is $\Delta \sigma=0.0125 \mu \mathrm{m}^{-1}$ with $\Delta \mathrm{L}=10 \mu \mathrm{m}$ the distance between grooves (corresponding to typical pixel pitch, not to the smallest distance that can be achieved between scattering centers). Indeed, the main issue is therefore not the Resolution (which is easily achievable with the cm length of the waveguide) but the spectral etendue, that is limited by matching of each scattering center with a pixel on top of the surface, which means limited by the pixel pitch $\Delta \mathrm{L}$.

Besides, in order to sample the stationary wave, instead of using nanodots of gold sputtered on the surface [7], or Focused Ion Beam technology (FIB) [8] we can use the waveguide's writing laser itself in order to realize hollow nanocylinders perpendicular to the waveguide and use the energy diffused by each cylinder to sample the optical intensity [11]:

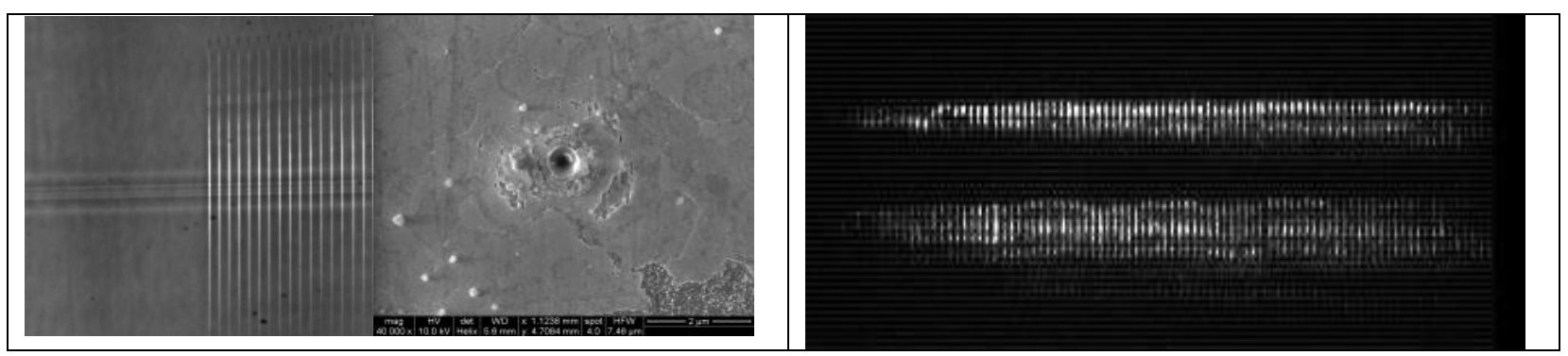

Fig. 4: from [11]: Left, image of the sampling cylinders obtained by photo-inscription (seen from above and seen from the side) Right, near IR image obtained in a Goodrich camera, showing the extracted signal achieved by the nano-cylinders, for two adjacent channel waveguides.

Our first concern was then to increase the spectral etendue, by multiplexing the SWIFTS concept: 


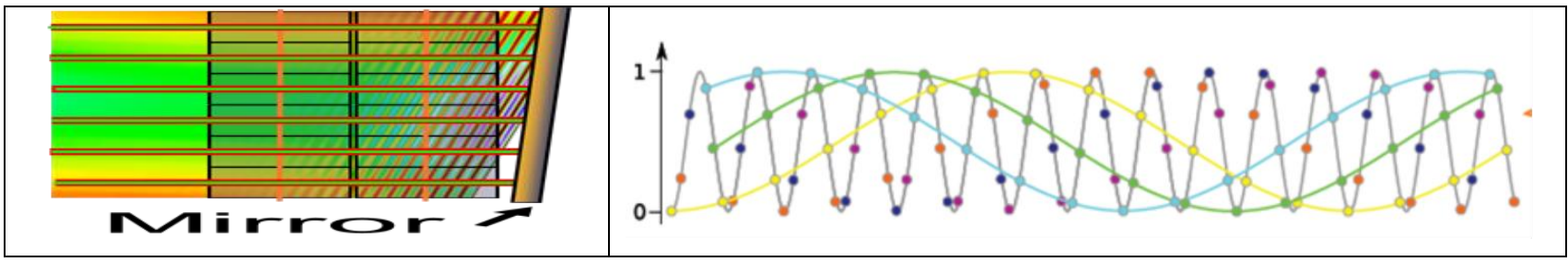

Fig. 4: SWIFTS WIDE concept. Left, parallel channel waveguides are coupled to a tilted mirror. The pixel matrix set on top of the sample will therefore detect a different part of the interference fringes. Right: Each individual waveguide undersamples the high frequency signal, resulting in Moiré fringes of large period. However, as the parallel waveguides are sampling different positions of

the interferogram, by superimposing accurately the shifted individual interferograms, the high frequency signal is reconstructed.

The idea is to fabricate several parallel channel waveguides into a substrate. Then, polishing the sample output and coating a metal layer (Ag, $\mathrm{Al})$ in order to obtain a tilted mirror. The detector matrix on top of the sample fixes the position of the scattering centers. The angle of tilt must be then calculated as a function of the number of channels: between the first and the last waveguide, tilt must ensure the sampling of the one period of the target spectrum, at its central wavelength. Our next objective was therefore to fabricate the lantern, that converts a multimode ( $\mathrm{N}$ modes) input, in a number $\mathrm{N}$ of single mode parallel waveguides.

\section{LASER WRITTEN LANTERNS}

As already discussed, the technique developed here, in collaboration with Univ. de Salamanca, was to induce Type I waveguides. We fabricated two types of lanterns: 1x4 and 1x16 lanterns, with different separations between channel waveguides, in order to adapt to the typical IR detector (Hamamatsu G12242-0707W InGaAs area image sensor) composed by $128 \times 128$ pixels arrayed at $20 \mu \mathrm{m}$ pitch. A first set was fabricated with $20 \mu \mathrm{m}$ pitch, to increase compacity and eventually increase the number of channels; and a second set was fabricated using $60 \mu \mathrm{m}$ pitch, in order to limit the eventual crosstalk between adjacent channels/pixels, by leaving a dark array of pixels between each sampled waveguide. The obtained lanterns, using optimized parameters ( 4 tracks with $2 \mu \mathrm{m}$ separation, $100 \mu \mathrm{m} / \mathrm{sec}$ and x50 objective) are shown in figure 5:

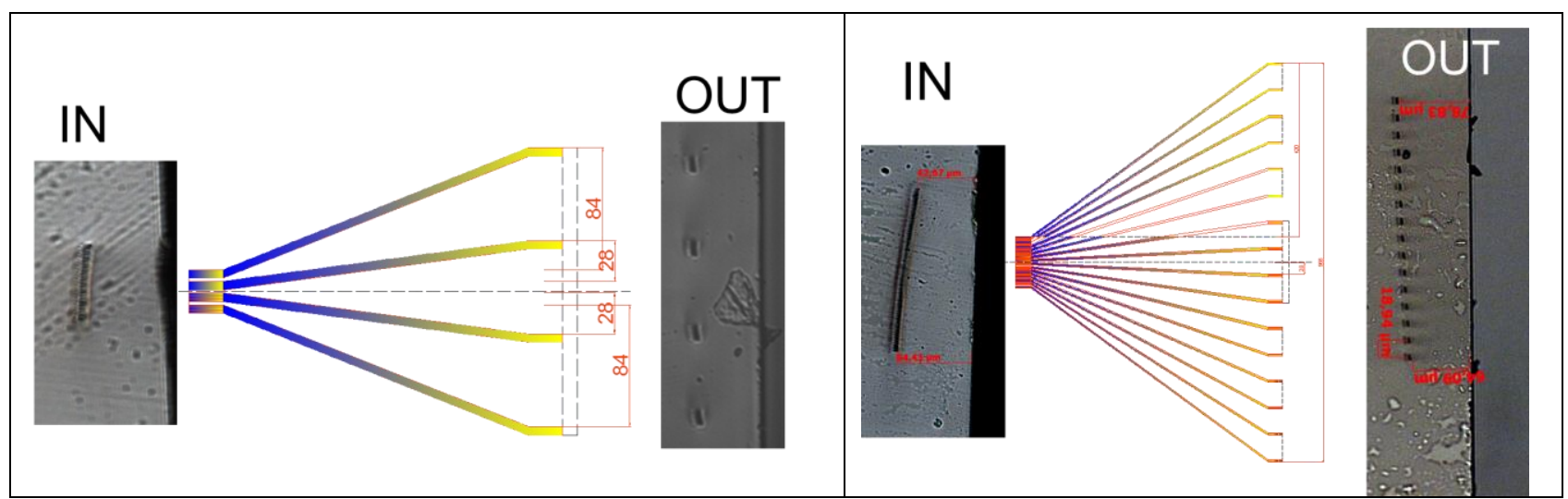

Fig. 5: Left, $1 \times 4$ lantern, where separation between channels is $60 \mu \mathrm{m}$. Right, $1 \times 16$ lantern, with $20 \mu \mathrm{m}$ separation. A second set with $1 \times 4$ and $20 \mu \mathrm{m}$ separation and $1 \times 16$ with $60 \mu \mathrm{m}$ separation was also fabricated (see fig. 6 ).

A preliminary observation of the outputs, using a 1580nm SLED injection, confirms that the system is confining light, but adapted injection (slit input) was not available at the time of this work, so simultaneous excitation of all the output channel was poorly obtained: 


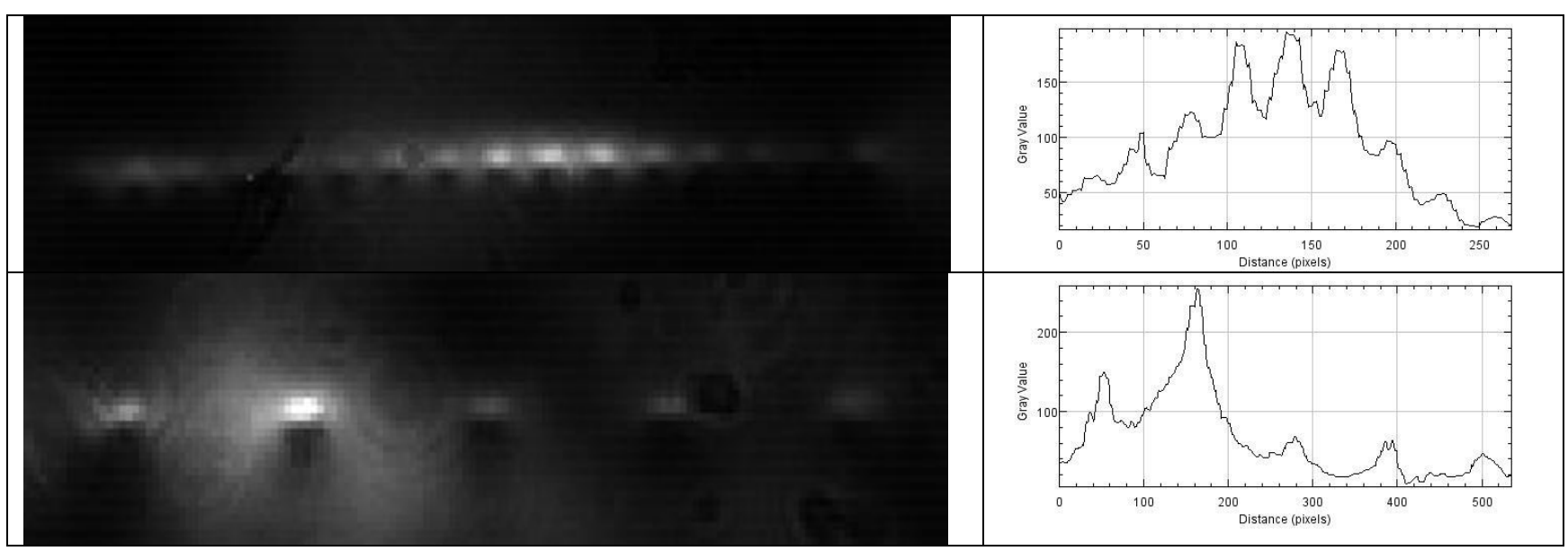

Fig. 6: Top: 1580nm image of the output 1x4 lantern, where separation between channels is $20 \mu \mathrm{m}$, at its profile on the right. Bottom: same observation, for the $1 \times 16$ lantern, with $60 \mu \mathrm{m}$ separation.

These preliminary results show that the simultaneous injection of several channels is obtained. The sample is not undergoing the tilted polishing and subsequent metal coating, in order to obtain the mirror. A complementary step of polishing the upper surface is needed in laser written waveguides, as the waveguides are $100 \mathrm{~mm}$ deep inside the substrate, otherwise the scattered signal diverges and crosstalk is observed. An alternative that we are currently studying (see paper 10701-28) is the possibility to directly write scattering antennas, that will reduce the scattering angle, therefore allowing for higher distances between the scattering center and the detector, and thus giving the possibility to keep the waveguides buried inside the material. Our next step was therefore to prepare the detector in order to get the active surface as close as possible to the sample.

\section{COUPLING WITH DETECTOR}

The idea of the compact spectrometer, is to get as close as possible the scattering centers with the pixels, in order to avoid crosstalk. In infrared detectors, this issue is critical, as the typical depletion zone is some hundreds of microns inside the detector surface. As already discusses, an approach using antennas is currently under study. In the meantime, we uncapsulated the Hamamatsu detector in order to get the pixels as close as possible to the physical surface:

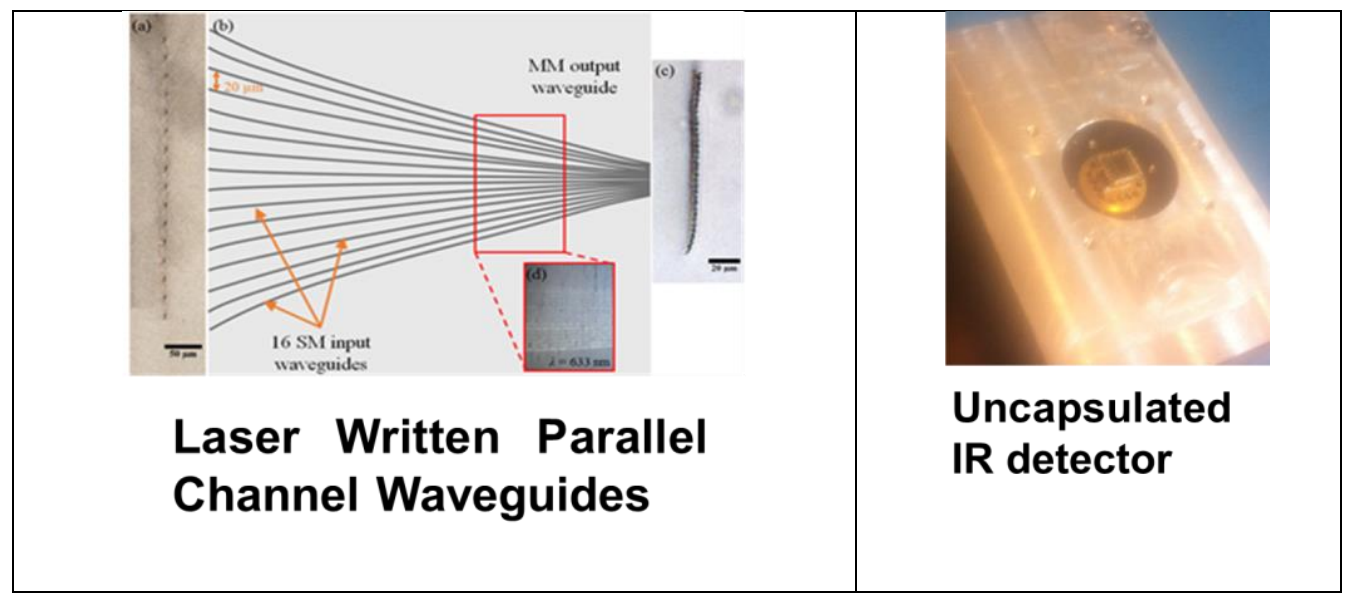


The first images of the detector on top of the channel waveguide (see paper 10701-28) confirmed that the system is still working, although we uncapsulated the detector. We have now to work of the gluing issues, to obtain a first compact prototype, combining the sample (parallel channel waveguides and scattering centers) and the detector.

\section{CONCLUSIONS \& PERSPECTIVES}

We have conceived, realized and characterized different laser written type I waveguides and lanterns in borosilicate glasses. We have validated the single mode behavior in the near IR $(1580 \mathrm{~nm})$ and obtained preliminary results on the simultaneous injection of parallel waveguides using a single multimode input IR lantern. The perspectives are now to polish the sample in order to get the waveguides close to the surface; to polish the output using a tilt angle, and coat with $\mathrm{Al}$ or $\mathrm{Ag}$ to obtain a mirror; and finally to couple the complete device to the uncapsulated Hamamatsu detector we have optimized.

\section{ACKNOWLEDGMENTS}

Authors acknowledge the funding from ASHRA (Action Specifique Haute Resolution Angulaire) from INSUCNRS.

\section{REFERENCES}

[1] Ródenas, A., Martin, G., Arezki, B., Psaila, N., Jose, G., Jha, A., Labadie, L., Kern, P., Kar, A., and Thomson, R. "Three-dimensional mid-infrared photonic circuits in chalcogenide glass" Optics Letters, Vol. 37, Issue 3, pp. 392394 (2012).

[2] Labadie, L., Martín, G., Anheier, N.C., Arezki, B., Qiao, H.A., Bernacki,B. and Kern, P. "First fringes with an integrated-optics beam combiner at $10 \mu \mathrm{m}$ A new step towards instrument miniaturization for mid-infrared interferometry" Astronomy\&Astrophysics 531, A48 (2011).

[3] Cheng, G., d'Amico, C., Liu, X., and Stoian, R., "Large mode area waveguides with polarization function by ultrafast laser photoinscription of a-SiO2" Opt. Lett. 38, 1924 (2013).

[4] F. Chen and J. R. Vazquez de Aldana, "Optical waveguides in crystalline dielectric materials produced by femtosecond-laser micromachining," Laser Photon. Rev. 8(2), 251-275 (2014).

[5] D. Choudhury, J. R. Macdonald, and A. K. Kar, "Ultrafast laser inscription: perspectives on future integrated applications," Laser Photonics Rev. 8(6), 827-846 (2014).

[6] T. Calmano and S. Müller, "Crystalline waveguide lasers in the visible and near-infrared spectral range," IEEE J. Sel. Top. Quantum Electron. 21(1), 1602213 (2015).

[7] E. Le Coarer, S. Blaize, P. Benech, I. Stefanon, A. Morand, G. L'erondel, G. Leblond, "Wavelength-scale stationary-wave integrated Fourier-transform spectrometry," Nature Phot. (London) 1,473-478 (2007).

[8] F. Thomas, S. Heidmann, M. de Mengin, N. Courjal, G. Ulliac, A. Morand, P. Benech, E. Le Coarer, and G. Martin "First Results in Near and Mid IR Lithium Niobate-Based Integrated Optics Interferometer Based on SWIFTSLippmann Concept"Journal of Lightwave Technology, Vol. 32, Iss. 22, pp. 3736-3742 (2014).

[9] Bonneville, Ch. et al, «SWIFTS : a groundbreaking integrated technology for high performances spectroscopy and optical sensors », SPIE MOEMS Photonics West, (2013)

[10] F. Thomas; M. De Mengin; C. Duchemin; E. Le Coarer; Ch. Bonneville; T. Gonthiez; A. Morand; P. Benech; J.-B. Dherbecourt; E. Hardy; E. Morino; R. Puget; B. Martin "High-performance high-speed spectrum analysis of laser sources with SWIFTS technology" Proc. SPIE 8992, Photonic Instrumentation Engineering, 89920I (8 March 2014).

[11] G. Martin, J. R. Vázquez de Aldana, A. Rodenas, C. d'Amico, R. Stoian, "Recent results on photonic devices made by laser writing: 3D 3T near IR waveguides, mid-IR spectrometers and electro-optic beam combiners," Proc. SPIE 9907, Optical and Infrared Interferometry and Imaging V, 990739 (26 July 2016) 